\title{
Retraction Note to: Nanoformulation of Apolipoprotein E3-Tagged Liposomal Nanoparticles for the Co-Delivery of KRAS-siRNA and Gemcitabine for Pancreatic Cancer Treatment
}

Fengyong Wang ' Zhen Zhang'

Published online: 1 February 2022

(C) Springer Science+Business Media, LLC, part of Springer Nature 2022

Retraction Note to: Pharmaceutical Research (2020) 37: 247

https://doi.org/ 10.1007/s I 1095-020-

02949-y

The Editor-in-Chief has retracted this article. The corresponding author requested retraction of the article because further work undertaken in their group after the publication of this study found that the data presented were not reproducible and therefore the conclusions presented are not reliable. Neither author responded to subsequent requests for comment on the retraction.

The online version of the original article can be found at https://doi.org/l 0 . | 007/s | | 095-020-02949-y

\section{Zhen Zhang}

zhezhang70@yahoo.com

Department of General Surgery, Tongde Hospital of Zhejiang Province, Hangzhou 310012 , Zhejiang Province, China 